\title{
THE ADDITION OF ARTESUNATE TO CHLOROQUINE FOR TREATMENT OF PLASMODIUM FALCIPARUM MALARIA IN GAMBIAN CHILDREN DELAYS, BUT DOES NOT PREVENT TREATMENT FAILURE
}

\author{
COLIN J. SUTHERLAND, CHRISTOPHER J. DRAKELEY, UCHE OBISIKE, ROSALIND COLEMAN, MUSA JAWARA, \\ GEOFFREY A. T. TARGETT, PAUL MILLIGAN, MARGARET PINDER, AND GIJS WALRAVEN \\ Department of Infectious and Tropical Diseases, London School of Hygiene and Tropical Medicine, London, United Kingdom; Joint \\ Malaria Programme, Moshi, Tanzania; Farafenni Field Station and Fajara Medical Research Council Laboratories, \\ Banjul, The Gambia
}

\begin{abstract}
In a randomized controlled trial, chloroquine monotherapy was compared with the combination of artesunate and chloroquine for treating uncomplicated Plasmodium falciparum malaria in 536 Gambian children. Chloroquine-treated children exhibited a 28 -day clinical failure rate of $15 \%(95 \%$ confidence interval $[\mathrm{CI}]=9.2-22 \%)$ compared with $11 \%(7.8-15 \%)$ among children receiving the combination $(P=0.08$, by Wilcoxon test $)$. Seventy-three percent of chloroquine-treated children exhibited parasitemia during follow-up compared with $49 \%$ of children receiving the combination (relative risk $=1.5,95 \% \mathrm{CI}=1.3-1.7 ; \chi^{2}=21.18, P<0.001$ ). A significant reduction in clinical and parasitologic treatment failure in the combination group occurred in the first two weeks following treatment, but this was eroded over weeks three and four of follow-up. The impact of combination therapy on the transmission of chloroquineresistant parasites is discussed. Chloroquine plus artesunate is not sufficiently efficacious to justify its introduction as a replacement for chloroquine monotherapy in The Gambia.
\end{abstract}

\section{INTRODUCTION}

Chloroquine $(\mathrm{CQ})$ remains the front-line treatment for uncomplicated Plasmodium falciparum malaria in much of Africa. In The Gambia in west Africa, evidence is accumulating that the prevalence of resistance to $\mathrm{CQ}$ is increasing. ${ }^{1-4} \mathrm{~A}$ change in treatment policy may be required soon because an increase in the prevalence of CQ-resistant $P$. falciparum infections has been linked to increasing malaria-related mortality among children in neighboring Senegal. ${ }^{5}$ The combination of sulfadoxine with pyrimethamine (SP) has been suggested as an affordable replacement drug for CQ. However, widespread use of SP has very rapidly resulted in the development of resistance among parasite populations in east Africa and in Malawi ${ }^{6,7}$ Thus, use of SP alone in The Gambia may provide only a short-term benefit.

In the context of increasing prevalence of $\mathrm{CQ}$ resistance in The Gambia, we have shown that treatment with CQ in 1998 preferentially selected for emergent gametocytes carrying resistance-associated alleles of the $P$. falciparum multidrug resistance gene 1 ( $p f m d r 1$ ) and the P. falciparum chloroquine resistance transporter ( $p f c r t)$ gene. ${ }^{4}$ The long-term public health benefit of using combination therapy in African settings will be realized only if such selective transmission is prevented. Therefore, drug combinations need to be evaluated both in terms of their efficacy/effectiveness and the transmission dynamics of parasites resistant to any of the component drugs.

One plausible strategy for sustainable therapeutic success for uncomplicated malaria cases is to introduce CQ plus artesunate (AS) as a combination therapy. This would leave SP, and other anti-folate combinations such as chlorproguanil/ dapsone, in reserve if required for the treatment of recrudescent infections. The combination of SP with AS has been shown to be highly efficacious in The Gambia ${ }^{8}$ and has the added benefit of significantly reducing post-treatment transmission compared with treatment with either $\mathrm{CQ}$ or SP alone. ${ }^{9}$ In this report, we evaluate the efficacy of $\mathrm{CQ}$ plus three daily doses of AS compared with CQ monotherapy as treatment for uncomplicated $P$. falciparum malaria in Gambian children.

\section{MATERIALS AND METHODS}

The study protocol was reviewed and approved by the Medical Research Council/Gambian Government Joint Ethical Committee, and the London School of Hygiene and Tropical Medicine Ethics Committee. Informed consent was obtained from the parents or guardians of all patients who participated in this study.

Study children and treatment. An established protocol for clinical trials with gametocyte carriage and transmission as the major endpoints ${ }^{9}$ was modified to measure additional end points of clinical failure, post-treatment parasite prevalence, and post-treatment parasite density. Children attending the clinic at Farafenni General Government Hospital in Farafenni, The Gambia were recruited from October 4 to December 5, 2000. Farafenni is a market town in a rural area $170 \mathrm{~km}$ from the Atlantic coast. Eligible children were 1-9 years old, lived within a radius of approximately $10 \mathrm{~km}$ of Farafenni (but not in nearby Senegal), had a body weight $>5 \mathrm{~kg}$, a history of fever, and $P$. falciparum asexual parasitemia $>500 / \mu \mathrm{L}$ of capillary blood. Eligible children were only recruited if a parent or guardian gave informed consent. Exclusion criteria included anemia (packed cell volume $[\mathrm{PCV}]<20 \%$ ), any signs or symptoms of severe malaria, inability to take drugs orally, treatment with any anti-malarial within the past two weeks, infection with either $P$. ovale or $P$. malariae, or any evidence of chronic disease or other acute infection.

Recruits were randomly assigned to receive three doses of CQ alone or CQ plus three doses of AS in a ratio of 1:3, using random numbers generated in Stata 6.0 (Stata Corp., College Station, TX). The study was designed primarily for transmission end points, and this ratio was chosen to give approximately equal gametocyte carriage rates at day $7 .^{9} \mathrm{~A}$ sample size of 500 was expected to provide sufficient statistical power to observe a difference in transmissibility of gametocytes arising after the two regimens. Study clinicians and field assistants 
(who administered treatment) were not blinded to the treatment given to each recruited child. However, the principal investigator, entomologist, and slide readers were unaware of the treatment group of each child until the conclusion of the study.

Children in the CQ treatment group were orally administered $25 \mathrm{mg}$ of CQ base (Alkaloida, Ltd., Tiszavasvari, Hungary) per kilogram of body weight over a three-day period. Children in the CQ plus AS group received $25 \mathrm{mg} / \mathrm{kg}$ of CQ base and $4 \mathrm{mg} / \mathrm{kg}$ of AS (Guilin Pharmaceutical Works, Guilin, People's Republic of China and supplied by Sanofi, Paris, France) daily for three days. No artesunate placebo was given to the children on CQ monotherapy. All drugs were prescribed by a study clinician who directly supervised the first dose. The second and third doses were supervised by a trained field assistant in the child's home. Children were observed for 30 minutes after the first dose, and any child who vomited was administered a replacement dose. Study numbers were not assigned until this first dose was successfully administered. Children who repeatedly vomited oral treatment were given $\mathrm{CQ}$ as an injection, or admitted to the pediatric ward of the hospital. These children were not assigned a study number, and the next eligible child was recruited in their place, with the same treatment. Each child also received $10 \mathrm{mg} / \mathrm{kg}$ of paracetamol under supervision in the recruitment clinic, and parents or guardians were given further doses of paracetamol and instructed to administer it every six hours until the child's symptoms had subsided. Parents or caretakers were asked to return to the clinic on any day if the child's condition deteriorated or showed reason for concern.

If at any time during the four-week follow-up period a parent or guardian reported that a child was unwell, the child was examined by a physician. If the child had a parasitemia and fever (axillary temperature $\geq 37.5^{\circ} \mathrm{C}$ ) and did not have other conditions that could explain the symptoms, the child was considered a clinical treatment failure and given SP (half a tablet for children weighing $\leq 10 \mathrm{~kg}$ and an additional quarter tablet for each 5-kg increase in body weight; each tablet contained $12.5 \mathrm{mg}$ of pyrimethamine and $250 \mathrm{mg}$ of sulfadoxine) as a rescue treatment.

Laboratory samples. Peripheral blood from a finger prick in the recruiting clinic provided samples for duplicate thick blood films, a hematocrit capillary tube, and an EDTAmicrotainer sample $(50-100 \mu \mathrm{L})$ from each patient prior to treatment (day 0). One thick film was stained rapidly using Field's method and 20 high-power microscopic fields were read immediately in the clinic for diagnosis. Second blood films were returned to the laboratory and allowed to dry for 24 hours. Thick films collected at all time points were stained with Giemsa and were read twice, each time by a different trained microscopist; 100 high-power fields were examined by each reader before a slide was declared negative. For all thick films in the study, parasite density per microliter of blood was calculated assuming one parasite per high-power field is equivalent to 500 parasites per $\mu \mathrm{L} .{ }^{10}$

Post-treatment follow-up. In addition to visiting on days 1 and 2 to administer antimalarial treatment, field assistants visited each child at home on post-recruitment days 3,14, and 28. On each occasion, a finger prick blood sample was taken and used to make a thick film for microscopy. The child or child's parents were asked whether there had been improvement in the patient's condition, and whether there had been any relapse of symptoms. If on any of these visits the field assistant thought there was evidence of continuing or relapsing clinical malaria, the child was asked to attend the clinic as soon as possible for appraisal by a clinician and further treatment if necessary.

On day 7 after treatment, all patients were collected from their homes and taken to the Medical Research Council Laboratories in Farafenni, where peripheral blood was obtained by finger prick for thick blood film preparation and PCV estimation. Thick blood films were stained with Field's stain, and 100 fields were immediately examined for the presence of trophozoites or gametocytes. Gametocyte-positive subjects were asked to donate venous blood for transmission studies. $^{9}$

Data analysis. All children with follow-up data were included in the primary analysis. Clinical failure rates were estimated using the Kaplan-Meier method. ${ }^{11}$ Treatment failure rates were compared using the Wilcoxon test. All data were double-entered into Epi-Info version 6.0 (Centers for Disease Control and Prevention, Atlanta, GA). In the case of microscopy data, both first and second readings were doubleentered and the files were merged before the two readings were compared. Discrepancies in parasite density $> \pm 40 \%$ were resolved by re-reading of the relevant thick films. Clinical and parasitologic data entered in Epi-Info were transferred to Stata 6.0 (Stata Corp.) for statistical analysis.

\section{RESULTS}

A profile of the study is shown in Figure 1. A total of 1,502 children with fever (axillary temperature $\geq 37.5^{\circ} \mathrm{C}$ ) were screened. Nine hundred sixty-six were not eligible for enrollment. The most common reasons for ineligibility were slide negativity $(\mathrm{n}=434)$, use of CQ in the previous two weeks $(\mathrm{n}=110)$, refusal to give consent $(\mathrm{n}=100), P$. falciparum parasite density $<500 / \mu \mathrm{L}(\mathrm{n}=59)$, living outside the study area $(\mathrm{n}=57)$, repeated vomiting of medicine $(\mathrm{n}=51)$ and a PVC $<20 \%(n=29)$. Five hundred thirty-six children were recruited, 136 and 400 in the two treatment groups, respectively (ratio $=1: 2.9$ ). The baseline characteristics of the two groups are shown in Table 1.

Of the 536 recruits, 21 children (7 and 14 in the CQ and $\mathrm{CQ} / \mathrm{AS}$ groups, respectively) were found by additional microscopy to have no detectable $P$. falciparum and were therefore incorrectly read by the microscopists during the rapid screening procedure in the recruiting clinic. Seven of them were positive for $P$. malariae or $P$. ovale. An additional 18 recruited children (3 and 15 in the monotherapy and combination groups, respectively) were found to be positive at recruitment for either $P$. malariae or $P$. ovale, in addition to $P$. falciparum. These 39 children have been included in the primary analyses and in the trial profile, but were excluded in a secondary analysis.

Three fully observed doses of antimalarials were documented for $95.0 \%$ of the recruited children. Twenty-one children withdrew from the study during the first 48 hours for one of the following reasons: the child left the area $(n=5)$, the guardian gave a false address $(\mathrm{n}=5)$, consent was withdrawn (particularly by the child's father) $(\mathrm{n}=5)$, the parents took the child for additional treatment elsewhere (these may have been treatment failures) $(n=2)$, the child died $(n=1)$, and 


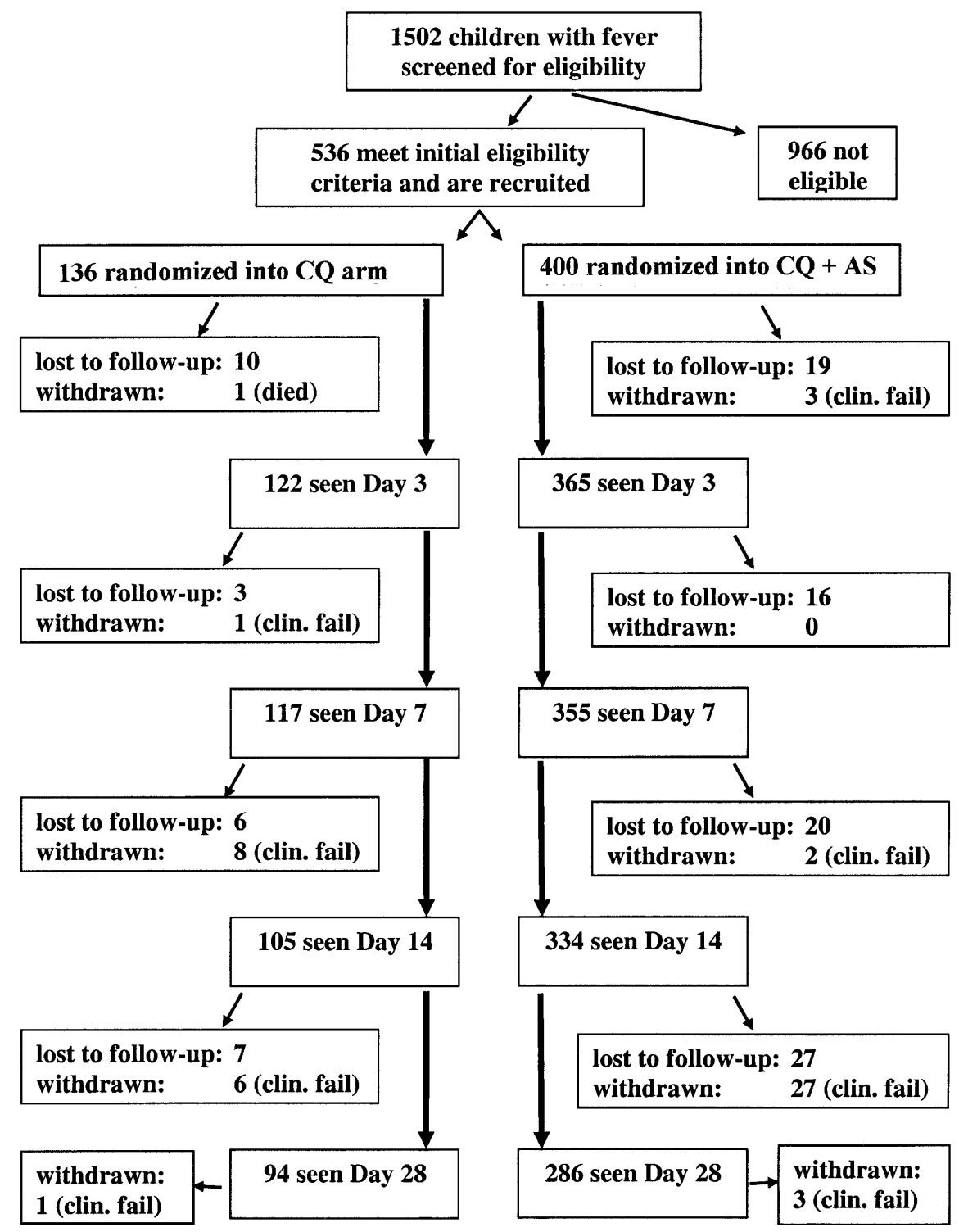

FIGURE 1. Study profile. $\mathrm{CQ}=$ chloroquine; $\mathrm{AS}=$ artesunate; clin. fail. = clinical failure.

other reasons $(n=3)$. None of these children was available for any subsequent follow-up visits. For six additional children, treatment data were not recorded on days 1 and 2; two of these children were among the 21 with no detectable $P$. falciparum parasitemia at recruitment. Thus, 472 recruited children (120 and 352 in the respective treatment groups) presented with a confirmed $P$. falciparum monoinfection and completed the treatment course under observation.
Six percent of children in each group were not seen on one of the scheduled follow-up days, but were seen on a later follow-up day. For example, some children were missed on day 7 , but were seen again on day 14 . These children have been included in the analysis and in the study profile.

Clinical treatment failure. One child in the CQ group (a two-year-old boy) died of malaria before completing full treatment and was treated in the analysis as an early treat-

TABLE 1

Characteristics of recruited patients at presentation*

\begin{tabular}{|c|c|c|}
\hline & $\begin{array}{l}\text { Chloroquine } \\
(\mathrm{n}=136)\end{array}$ & $\begin{array}{l}\text { Chloroquine plus artesunate } \\
\qquad(\mathrm{n}=400)\end{array}$ \\
\hline Age, years, mean (SD) & $4.9(2.32)$ & $4.9(2.29)$ \\
\hline PCV, mean packed cell, volume, \% (SD) & $31.4(5.36)$ & $31.4(5.06)$ \\
\hline Plasmodium falciparum density, mean $\log / \mu \mathrm{L}(95 \% \mathrm{CI})$ & $10.1(6.21-13.19)$ & $10.3(6.21-13.0)$ \\
\hline Temperature $\left({ }^{\circ} \mathrm{C}\right)$, mean $(95 \% \mathrm{CI})$ & $37.9(37.67-38.12)$ & $37.9(37.75-38.02)$ \\
\hline$\%$ monthly recruitment (Oct:Nov:Dec) & $(36.7: 54.4: 8.8)$ & $(44.0: 49.0: 7.0)$ \\
\hline Presence of gametocytes (prevalence) & $20.6 \%$ & $20.0 \%$ \\
\hline
\end{tabular}

\footnotetext{
$* \mathrm{PCV}=$ packed cell volume; $\mathrm{CI}=$ confidence interval.
} 
ment failure. This child satisfied all inclusion criteria and was recruited on a Monday around noon. He received the second dose at home on Tuesday morning, but his condition rapidly deteriorated. He was taken by his family to the pediatric ward of Farafenni Hospital where he died on Tuesday night. Of those children not lost to follow-up, there were no other deaths of recruited children.

Of the 536 children recruited into the study, treatment outcome was known for $428(80 \%)$. At any time over the four weeks of follow-up, CQ-treated children were more likely to experience treatment failure than children who received CQ plus AS (Figure 2), but this difference was not significant at the $5 \%$ level. Fifty-two children were found during the fourweek follow-up period to have a continuation or recurrence of clinical malaria, $17(12.5 \%)$ and $35(8.8 \%)$ children enrolled in the $\mathrm{CQ}$ and $\mathrm{CQ} / \mathrm{AS}$ groups, respectively. An additional five children ( 4 and 1 in the $\mathrm{CQ}$ and CQ/AS groups, respectively) harbored parasites on day 3 at $\geq 25 \%$ of the pre-treatment parasite density on day $0,{ }^{12}$ but were not considered clinical treatment failures under our protocol. Kaplan-Meier estimates of the treatment failure rates were $15 \%$ $(95 \%$ confidence interval $[\mathrm{CI}]=9.2-22 \%)$ and $11 \%$ $(7.8-15 \%)$, respectively $\left(\chi^{2}=3.03, P=0.08\right.$, by Wilcoxon test for equality of survivor functions). Finger prick blood samples taken at the first follow-up visit after rescuetreatment with SP were available for 21 of these children. Seventeen of these 21 children ( $81 \%$ ) were successfully cleared of asexual parasites, while the remaining four children had persistent asexual parasitemia following administration of SP, suggesting further (asymptomatic) treatment failure.

Although the combination provided a benefit in terms of increased treatment success, this benefit was transient, and was seriously eroded between day 14 and day 28 (Figure 2). In fact, a highly significant difference in clinical failure rates would have been observed if the study had been terminated at
14 days because prior to day 15 of follow-up there were 13 clinical failures among CQ-treated children (Kaplan-Meier estimate $=11 \%, 95 \% \mathrm{CI}=6.5-18 \%)$ and only seven among CQ/AS-treated children $\left(2 \%, 95 \%\right.$ CI $=0.9-4.1 \% ; \chi^{2}=$ 17.4, $P<0.001$ ) (Figure 2).

Age and clinical failure. In both treatment groups, treatment failure was significantly higher among children less than five years old (hazard ratio $=1.8,95 \% \mathrm{CI}=1.04-3.2, P=$ 0.035) (Table 2). Treatment failure rates were also higher among children treated in October, the first month of recruitment, than in November/December (hazard ratio $=1.8,95 \%$ $\mathrm{CI}=1.01-3.1, P=0.045)$.

Parasitologic treatment failure. Children with a thick film positive for P. falciparum trophozoites in the absence of fever or other clinical signs of malaria during follow-up were considered asymptomatic parasitologic treatment failures and were not given SP as rescue treatment. Parasitologic analysis was restricted to children known to have completed the treatment course that had presented at enrollment with a confirmed $P$. falciparum infection of at least 500 parasites $/ \mu \mathrm{L}$ ( $\mathrm{n}=472,120$ and 352 in the CQ and CQ/AS groups, respectively). We chose this "on-treatment" approach specifically to evaluate the parasite-killing effect of the full three doses of AS.

The prevalence of parasitemia during the follow-up period is shown for both treatment groups in Figure 3. At each time point, the prevalence of trophozoites was significantly higher among children treated with $\mathrm{CQ}$ than among those treated with CQ/AS $\left(\chi^{2}=92.98,40.09,21.16\right.$, and 12.67, respectively, $P<0.001$ in each case). Among fully treated children, $49.1 \%$ in the combination group and $73.3 \%$ in the monotherapy group experienced at least one episode of parasitemia, with or without symptoms of clinical malaria (relative risk $=1.49$, $95 \%$ CI $\left.=1.28-1.74, \chi^{2}=21.18, P<0.001\right)$. Thus, the combination confers a significant benefit in reducing carriage of

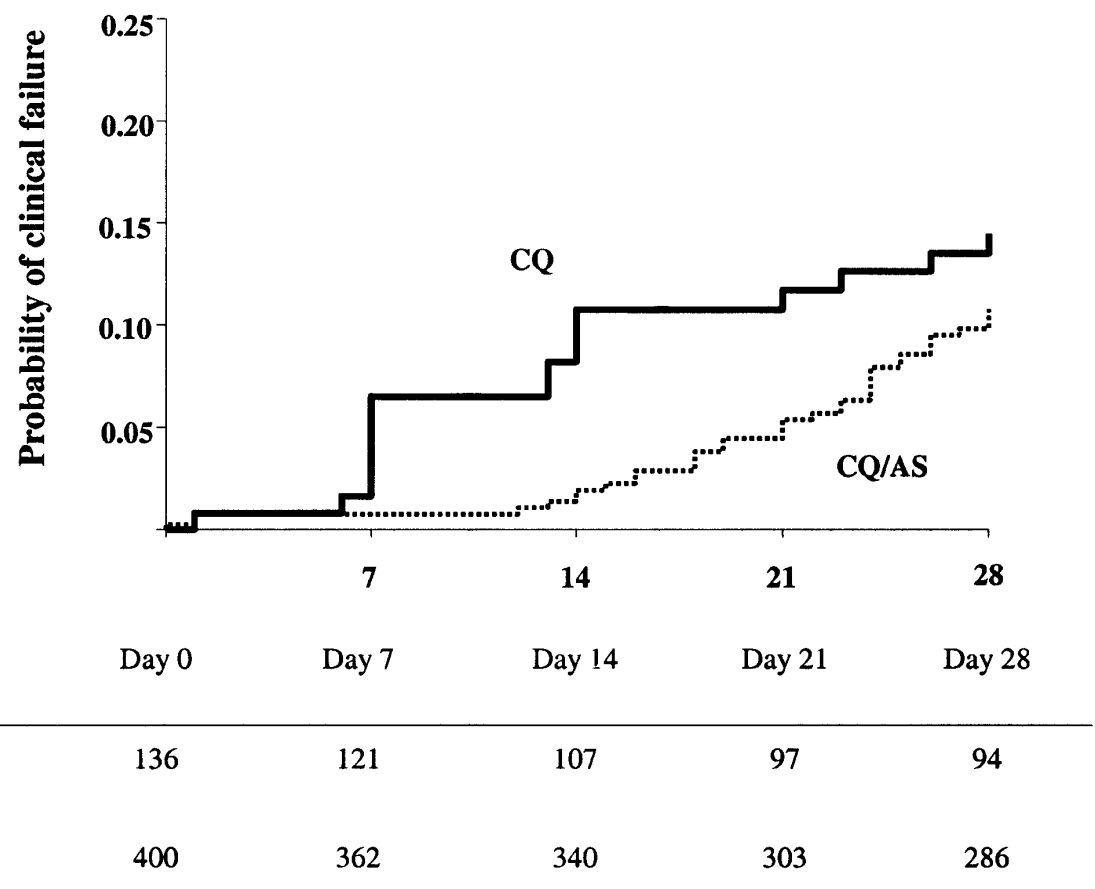

FIGURE 2. Kaplan-Meier estimates of clinical failure rates after treatment with chloroquine (CQ) and CQ plus artesunate (AS). The plots represent numbers of children at risk in each group at each time point. 
TABLE 2

Kaplan-Meier estimates of clinical failure rates $(95 \% \mathrm{CI})$ with age stratification*

\begin{tabular}{llcc}
\hline & & CQ & CQ plus AS \\
\hline Day 14 & $<5$ years old & $15 \%(8.0,2.7)$ & $3.2 \%(1.3,7.5)$ \\
& $\geq 5$ years old & $6.8 \%(2.6,17)$ & $1 \%(0.3,4.0)$ \\
Day 28 & $<5$ years old & $19 \%(11,31)$ & $15 \%(10,22)$ \\
& $\geq 5$ years old & $10 \%(5,22)$ & $7.8 \%(5,13)$ \\
\hline \multirow{2}{*}{ * CI = confidence interval; CQ $=$ chloroquine; AS = artesunate. }
\end{tabular}

parasites after treatment compared with monotherapy, albeit with a cumulative anti-parasite efficacy over 28 days of only $51 \%$.

Trophozoite density among parasite-positive subjects increased from day 3 during follow-up in both treatment groups (Figure 4). The mean natural logarithm (ln) of the parasite density was significantly lower in the combination group on days 3,7 , and 14 . The significant reduction in parasite density seen in the first week post-treatment among children treated with the combination was partially eroded by day 14 , and by day 28 there was no difference in mean ln parasite density between the treatment groups (Figure 4). This is consistent with the hypothesis that parasitemia occurring late in followup is largely derived from the expansion of low or undetectable populations of parasites that survive treatment. ${ }^{4}$

Since parasite densities are affected by immune responses of the host, age may be expected to have an effect on this parameter. For example, presentation parasitemia was on average higher among children less than five years old, and this difference was significant at the $10 \%$ level. Among children harboring trophozoites during follow-up, there was a similar small effect of age on parasite density at day 7. Children less than five years old who were parasite positive had an average density of $3,777(95 \% \mathrm{CI}= \pm 2,340)$ parasites $/ \mu \mathrm{L}$ at day 7 , whereas for those more than five years old, the average den-

\section{Prevalence of post-treatment trophozoites}

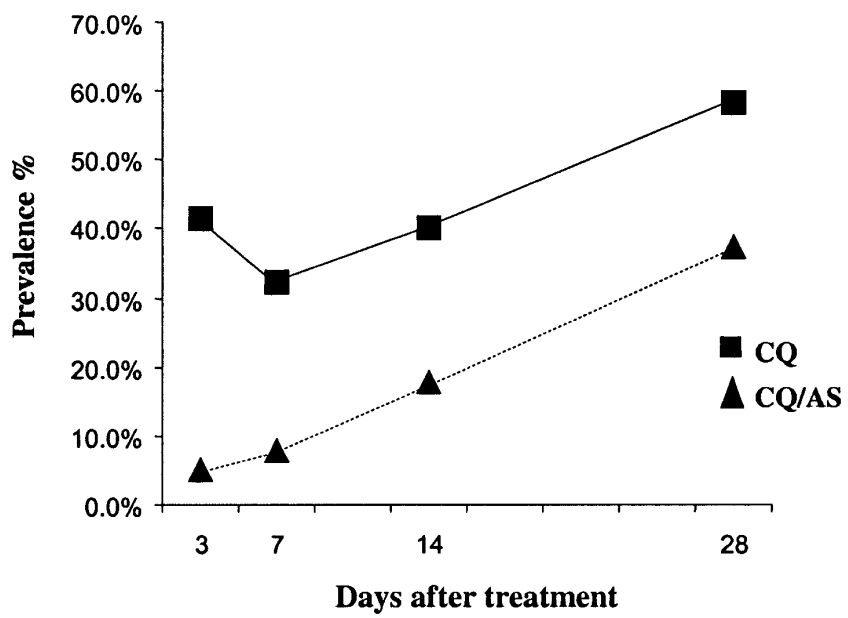

Figure 3. Prevalence of Plasmodium falciparum trophozoites in fully treated children at each of the four time points following treatment with either chloroquine (CQ) or CQ/artesunate (AS). The denominators are Day 3, 113 and 338 children, respectively, in the monotherapy and combination groups; Day 7, 108 and 326 children; Day 14, 102 and 307 children, and Day 28, 96 and 280 children. sity was $1,966(95 \% \mathrm{CI}= \pm 1,544)$ parasites $/ \mu \mathrm{L}(\mathrm{P}=0.101$, by one-sided $t$-test). There was no discernable effect of age on parasite density at any other point in follow-up.

\section{DISCUSSION}

We have shown that Gambian children with uncomplicated falciparum malaria who receive a three-day regimen of $\mathrm{CQ}$ plus AS exhibit a clinical failure rate of $11 \%$ and a cumulative parasitologic failure rate of $52.1 \%$ over a 28 -day period. This regimen is thus much less efficacious in the Farafenni area than is a three-day regimen of AS combined with a single dose of SP, which has a treatment success rate of more than $97 \%$ in The Gambia. ${ }^{8,13}$ Although CQ/AS does provide a transient benefit in clinical efficacy over CQ monotherapy (Figure 2), a transient benefit in reducing parasite density (Figure 4), and a more robust benefit in reducing the prevalence of post-treatment asexual parasitemia (Figure 3), we conclude that $\mathrm{CQ} / \mathrm{AS}$ would not be a suitable choice to replace CQ as the first-line antimalarial regimen in the Gambia.

The poor efficacy of the combination is likely to be caused by the moderately high prevalence of CQ-resistant parasites in the study area. ${ }^{4}$ Studies carried out in 1998 found that the parasitologic efficacy of CQ over a 28-day period was approximately $70 \%{ }^{13}$ The results presented herein clearly show that two years later, only $25 \%$ of the CQ-treated children did not have an episode of post-treatment parasitemia, which suggests that the prevalence of resistant parasites has increased markedly. Artesunate is extremely effective at rapidly reducing parasite biomass, but only for a matter of hours after being administered and a seven-dose course of AS monotherapy has been considered necessary to effect radical cure. ${ }^{14,15}$ Thus, a small number of parasites can escape the three-dose regimen in some patients, as we have shown recently using a reverse transcriptase-polymerase chain reaction. ${ }^{16}$ It follows that in a patient population with a significant prevalence of CQ-resistant parasites, a proportion of these will be able to recrudesce because of their resistance to CQ, which persists at effective levels in the blood for six weeks. ${ }^{17}$ It is therefore very unlikely that replacement of CQ monotherapy with the $\mathrm{CQ} / \mathrm{AS}$ combination would significantly slow the increasing prevalence and distribution of CQ-resistant parasites in The Gambia.

A striking feature of our data is that, compared with $\mathrm{CQ}$ monotherapy, $\mathrm{CQ} / \mathrm{AS}$ reduces the proportion of early clinical treatment failures, but that over a 28-day follow-up period there is little overall reduction in failures (Figure 2). A similar transient benefit of the combination compared with $\mathrm{CQ}$ alone is seen in the first week as a significant reduction of mean positive parasite density among those children treated with $\mathrm{CQ} / \mathrm{AS}$. By day 14, this difference remains significant but has decreased, and it has disappeared by day 28 (Figure 4). Thus, if we had used a standard 14-day protocol to compare the sensitivity of our parasite population to the two regimens used, ${ }^{12}$ we would have concluded that CQ/AS confers a significant reduction in the proportion of clinical treatment failures and in mean positive parasite density. Following patients to day 28 demonstrates that these benefits are transient, and that re-treatment is eventually needed for a substantial number of children with malaria who are treated with $\mathrm{CQ} / \mathrm{AS}$. These findings are consistent with the results from two recent 


\section{Mean log trophozoite densities}

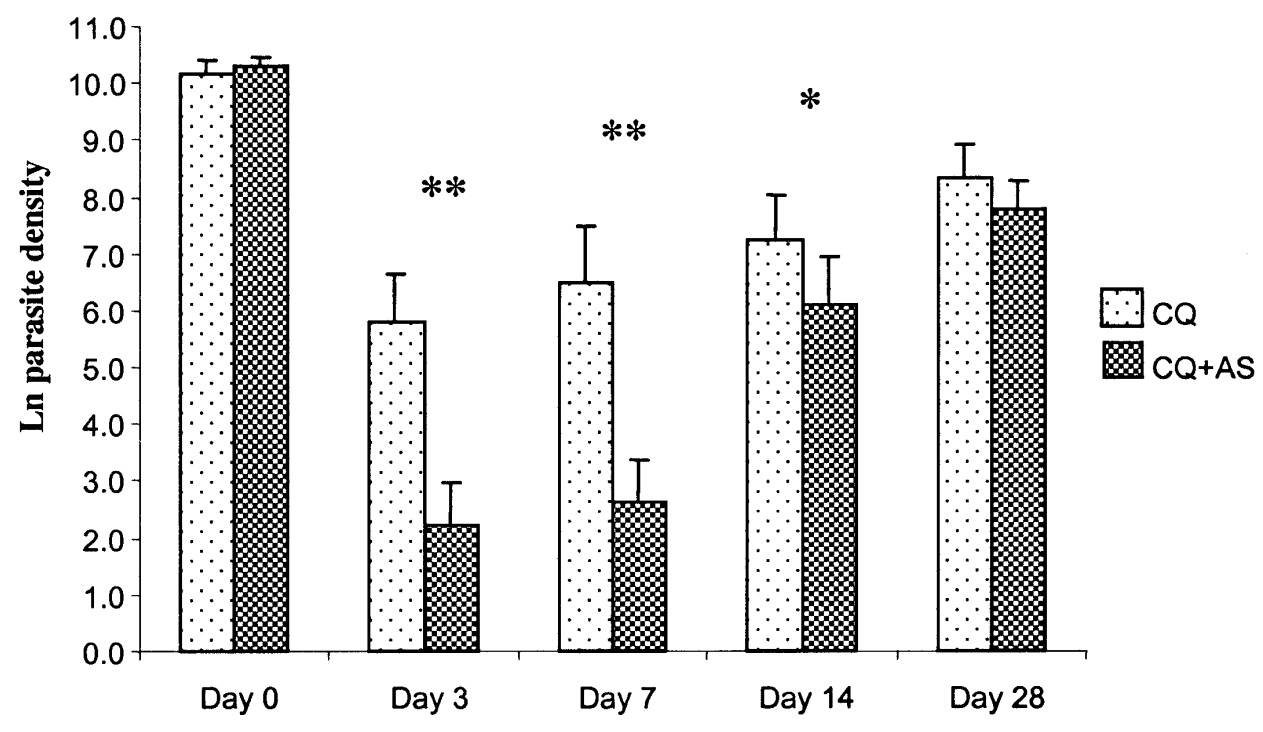

Days after treatment

FIGURE 4. Mean natural logarithm (ln) trophozoite density in children who were fully treated with either chloroquine (CQ) or CQ/artesunate (AS). The mean of the natural logarithm of parasite density among slide-positive children is shown for each time point. Bars show the $95 \%$ confidence intervals of the mean. Differences between means were tested by the Student's $t$-test. *significance at the $5 \%$ level; **significance at the $1 \%$ level.

studies using other drug combinations. Adjuik and others ${ }^{18}$ used a 28-day design to evaluate the relative efficacy of amodiaquine versus amodiaquine/AS, and found lower cure rates at day 28 than at day 14. Dorsey and others ${ }^{19}$ obtained a similar result in Uganda when treatment with SP was compared with SP plus AS. There, the best combination was SP plus amodiaquine. We would argue that longer follow-up provides a much more accurate picture of how well drugs work in the field, and that study protocols should always include follow-up beyond the two-week mark especially when combinations include drugs with long half-lives.

The transient nature of the benefit of the CQ/AS combination is consistent with the hypothesis that among CQtreated children in the Farafenni area, most or all posttreatment parasitemia is caused by recrudescent parasites rather than reinfection. We have argued that the crucial difference between RI, RII, and RIII patterns of recrudescence in CQ-treated children is the proportion of the original infection made up of CQ-resistant parasites. ${ }^{4}$ If this proportion is very low, and thus below the level of detection by microscopy, the patient will appear to clear the infection immediately following treatment, and any subsequent parasitemia arising from the expansion of this small remnant will appear as an RI type, or perhaps be considered a new infection, even with molecular strain typing.

Among parasites exposed to the combination, we envisage that the vast majority of both CQ-resistant and CQ-sensitive genotypes will be killed in the hours that follow administration of each dose of AS. The chance of a recrudescence occurring will then depend on the proportion of CQ-resistant parasites among the remnant that escape these three doses of AS. Therefore, we would predict that recrudesence of resis- tant parasites among combination-treated patients will take longer to reach patency and occur less frequently than among patients treated with CQ monotherapy. This is what we have observed. We are currently undertaking a comprehensive genotype analysis of parasite DNA collected from this study to test this hypothesis. Preliminary genotyping experiments with 20 parasite isolates from children in the CQ/AS group that were thick-film-positive on day 14 found that 19 of the children harbored parasites with CQ resistance-associated alleles at the pfcrt locus (Ord R and Sutherland CJ, unpublished data). ${ }^{4}$ This suggests that gametocytes emerging after treatment with the combination may also be selected for CQ resistance, as we have previously shown among gametocytes emerging after CQ monotherapy. ${ }^{4}$

Our results show that one highly effective antimalarial (AS) plus one relatively ineffective antimalarial (CQ) equals a poor combination. However, in contrast, the addition of AS to vulnerable and failing mefloquine in northern Thailand proved highly efficacious, and contributed to a significant reduction in gametocyte carriage rates. ${ }^{20}$ The Thai study population may not be comparable to African settings such as The Gambia because few exposed people have acquired immunity against malaria and asymptomatic infections are very rare. Nevertheless, it is simplistic to regard drug combinations as simply additive; important interactions between two drugs may occur on a number of levels including cross-resistance, inverse selection where one drug selects for a genotype conferring sensitivity to the other drug, and antagonism. ${ }^{21,22}$ Thus, empirical testing of apparently rational combinations in different endemic settings is essential to determine which are appropriate in each of those settings.

In conclusion, we have shown that the combination $\mathrm{CQ} / \mathrm{AS}$ 
is not sufficiently efficacious to justify its introduction as a first-line treatment regimen for malaria in The Gambia, despite it having some measurable benefits over CQ monotherapy. Our evidence also suggests that use of the combination would do little to reduce the transmission of CQ-resistant parasites in our study area. The major reason for the poor efficacy of this combination in the Farafenni area appears to be the already moderate-to-high prevalence of CQ-resistant $P$. falciparum infections. Therefore, this combination may yet prove to be useful against $P$. falciparum in settings where CQ resistance is rare, or in settings where $P$. vivax is the major malaria pathogen and sensitivity to $C Q$ remains high. This precautionary measure may prevent the emergence or further selection of CQ-resistant vivax malaria.

Received January 25, 2003. Accepted for publication April 2, 2003.

Acknowledgments: We thank Professor Keith McAdam for his support, and Dr. Neal Alexander for helpful discussions. We acknowledge the crucial contributions of the field and laboratory staff of the Farafenni Field Station and of the nursing staff of the Gambian Government Hospital in Farafenni. We particularly thank the children and parents from Farafenni who participated in the study.

Financial support: This study was supported by Wellcome Trust Project no. 061910 (awarded to Colin J. Sutherland) and by the Medical Research Council Laboratories, The Gambia (project no. 838).

Authors' addresses: Colin J. Sutherland and Geoffrey A.T. Targett, Department of Infectious and Tropical Diseases, London School of Hygiene and Tropical Medicine, Keppel Street, London, WC1E 7HT, United Kingdom, Telephone: 44-020-7927-2338, Fax: 44-20-76368739, E-mail: colin.sutherland@1shtm.ac.uk. Christopher J. Drakeley, Joint Malaria Programme, PO Box 2228, Moshi, Tanzania. Uche Obisike, Rosalind Coleman, Musa Jawara, and Gijs Walraven, Farafenni Field Station, Farafenni, The Gambia. Paul Milligan Margaret Pinder, Fajara Medical Research Council Laboratories, PO Box 273, Banjul, The Gambia.

\section{REFERENCES}

1. Menon A, Otoo LN, Herbage EA, Greenwood BM, 1990. A national survey of the prevalence of chloroquine resistant Plasmodium falciparum malaria in The Gambia. Trans $R$ Soc Trop Med Hyg 84: 638-640.

2. von Seidlein L, Duraisingh,MT, Drakeley CJ, Bailey R, Greenwood BM, Pinder M,1997. Polymorphism of the Pfmdr1 gene and chloroquine resistance in Plasmodium falciparum in The Gambia. Trans $R$ Soc Trop Med Hyg 91: 450-453.

3. Muller O, Boele van Hensbroek M, Jaffar S, Drakeley C, Okorie C, Joof D, Pinder M, Greenwood B, 1996. A randomised trial of chloroquine, amodiaquine and pyrimethamine-sulphadoxine in Gambian children with uncomplicated malaria. Trop Med Int Health 1: 124-132.

4. Sutherland CJ, Alloueche A, Curtis J, Drakeley CJ, Ord R, Duraisingh M, Greenwood BM, Pinder M, Warhurst DC, Targett GA, 2002. Gambian children successfully treated with chloroquine can harbor and transmit Plasmodium falciparum gametocytes carrying resistance genes. Am J Trop Med Hyg 67: 578-585.

5. Trape JF, Pison G, Preziosi MP, Enel C, Desgrees du Lou A, Delaunay V, Samb B, Lagarde E, Molez JF, Simondon F, 1998. Impact of chloroquine resistance on malaria mortality. $C R$ Acad Sci III 321: 689-697.

6. Ronn AM, Msangeni HA, Mhina J, Wernsdorfer WH, Bygbjerg IC, 1996. High level of resistance of Plasmodium falciparum to sulfadoxine-pyrimethamine in children in Tanzania. Trans $R$ Soc Trop Med Hyg 90: 179-181.
7. Nwanyanwu OC, Ziba C, MacHeso A, Kazembe P, 2000. Efficacy of sulphadoxine-pyrimethamine for acute uncomplicated malaria due to Plasmodium falciparum in Malawian children under five years old. Trop Med Int Health 5: 355-358.

8. von Seidlein L, Milligan P, Pinder M, Bojang K, Anyalebechi C, Gosling R, Coleman R, Ude JI, Sadiq A, Duraisingh M, Warhurst D, Alloueche A, Targett G, McAdam K, Greenwood B, Walraven G, Olliaro P, Doherty T, 2000. Efficacy of artesunate plus pyrimethamine-sulphadoxine for uncomplicated malaria in Gambian children: a double-blind, randomised, controlled trial. Lancet. 355: 352-357.

9. Targett G, Drakeley C, Jawara M, von Seidlein L, Coleman R, Deen J, Pinder M. Doherty T, Sutherland C, Walraven G, Milligan P, 2001. Artesunate reduces but does not prevent posttreatment transmission of Plasmodium falciparum to Anopheles gambiae. J Infect Dis 183: 1254-1259.

10. Greenwood BM, Armstrong JRM, 1991. Comparison of two simple methods for determining malaria parasite density. Trans R Soc Trop Med Hyg 85: 186-188.

11. Collet D, 1994. Modelling Survival Data in Medical Research. London: Chapman and Hall.

12. WHO, 1999. A Practical Handbook for Antimalarial Drug Therapeutic Efficacy Testing for the District Health Worker. WHO Regional Office for Africa. Geneva: World Health Organization.

13. von Seidlein L, Jawara M, Coleman R, Doherty T, Walraven G, Targett G, 2001. Parasitaemia and gametocytaemia after treatment with chloroquine, pyrimethamine/sulfadoxine, and pyrimethamine/sulfadoxine combined with artesunate in young Gambians with uncomplicated malaria. Trop Med Int Health 6: 92-98.

14. White NJ, 1994. Clinical pharmacokinetics and pharmacodynamics of artemisinin and derivatives. Trans $R$ Soc Trop Med Hyg 88 (Suppl 1): S41-S43.

15. Hoshen MB, Na-Bangchang K, Stein WD, Ginsburg H, 2000. Mathematical modelling of the chemotherapy of Plasmodium falciparum malaria with artesunate: postulation of 'dormancy', a partial cytostatic effect of its drug, and its implication for treatment regimens. Parasitology 121: 237-246.

16. Sutherland CJ, Alloueche A, McRobert L, Ord R, Leggat J, Snounou G, Pinder M, Targett GAT, 2002. Genetic complexity of Plasmodium falciparum gametocytes isolated from the peripheral blood of treated Gambian children. Am J Trop Med Hyg 66: 700-705.

17. Dollery C, ed., 1999. Therapeutic Drugs. Second edition. London: Churchill Livingstone.

18. Adjuik M, Agnamey P, Babiker A, Borrmann S, Brasseur P, Cisse M, Cobelens F, Diallo S, Faucher JF, Garner P, Gikunda S, Kremsner PG, Krishna S, Lell B, Loolpapit M, Matsiegui PB, Missinou MA, Mwanza J, Ntoumi F, Olliaro P, Osimbo P, Rezbach P, Some E, Taylor WR, 2002. Amodiaquineartesunate versus amodiaquine for uncomplicated Plasmodium falciparum malaria in African children: a randomised, multicentre trial. Lancet 359: 1365-1372.

19. Dorsey G, Njama D, Kamya M, Cattamanchi A, Kyabayinze D, Staedke S, Gasasira A, Rosenthal P, 2002. Sulphadoxine/ pyrimethamine alone or with amodiaquine or artesunate for treatment of uncomplicated malaria: a longitudinal randomized trial. Lancet 360: 2031-2038.

20. Price R, Nosten F, Luxemburger C, Kuile FOT, Paiphun L, Chongsuphajaisiddhi T, White NJ, 1996. Effects of artemisinin derivatives on malaria transmissibility. Lancet 347: 1654-1658.

21. Fivelman QL, Walden JC, Smith PJ, Folb PI, Barnes KI, 1999. The effect of artesunate combined with standard antimalarials against chloroquine-sensitive and chloroquine-resistant strains of Plasmodium falciparum in vitro. Trans $R$ Soc Trop Med Hyg 93: 429-432.

22. Warhurst DC, Duraisingh MT, 2001. Rational use of drugs against Plasmodium falciparum. Trans $R$ Soc Trop Med Hyg 95: 345-346. 